\title{
How 'learnable' are CASE tools in diverse user communities?
}

\author{
Brenda Scholtz ${ }^{1}$ and Janet Wesson ${ }^{2}$ \\ Department of Computer Science and Information Systems (CS \& IS), Nelson \\ Mandela Metropolitan University, P O Box 77000, Port Elizabeth, 6061, South \\ Africa \\ ${ }^{1}$ brenda.scholtz@nmmu.ac.za \\ 2 janet.wesson@nmmu.ac.za
}

\begin{abstract}
The use of Computer Aided Software Engineering (CASE) tools for teaching object-oriented systems analysis and design (OOSAD) and the Unified Modelling Language (UML) has many potential benefits, but there are several problems associated with the usability and learnability of these tools. This paper describes a study undertaken to determine if computing students from a linguistically and technologically diverse community experience problems with learning to use a CASE tool, and to determine if there is a relationship between two user characteristics of the students and the learnability of CASE tools.
\end{abstract}

Keywords: CASE tool, usability evaluation, learnability, UML, language diversity

\section{Introduction}

The Unified Modelling Language (UML) has been made an OMG (Object Management Group) standard (Frosch-Wilke, 2003) and helps analysts specify, visualise and document models of software systems, and thus improve the chances of success of the project (Kemerer, 1992; Brewer and Lorenz, 2003). The use of Computer Aided Software Engineering (CASE) tools for teaching UML has a number of potential benefits but there are several problems associated with the usability and learnability of these tools (Lending and Chervany, 1998; Booch et al., 1999; Post and Kagan, 2000; Burton and Bruhn, 2004). Learnability is one of 
the most important attributes of usability and refers to the capability of the system to enable the user to learn to use the application (Nielsen, 1993).

Some guidelines are available relating to the usability and learnability of UML and CASE tools (Jarzabek and Huang, 1998; Booch et al., 1999; Johnson and Wilkinson, 2003), however there is a lack of guidance regarding what is necessary to make a CASE tool 'learnable' and how to evaluate the learnability attribute of usability (Phillips et al., 1998). By performing CASE tool learnability evaluations, knowledge of the factors that influence the rate of learning can be determined, which can lead to improved approaches to teaching of CASE tools.

The South African university education system serves students from a wide range of backgrounds (Rauchas et al., 2006). There is enormous language diversity, with the country having 11 recognised official languages. The student community at a South African university can therefore be classified as linguistically diverse (Koch, 2002; Greyling and Calitz, 2003). Research has shown that the non-English speaking students are disadvantaged (Koch, 2002). These student communities have different frequency of computer user profiles and are thus technologically diverse. The home language and frequency of computer use are thus two important user characteristics for South African university students, and could affect the rate at which they learn to use a CASE tool.

The structure of the rest of the paper is as follows. Section 2 gives an overview of the concepts of usability and learnability, and explains Senapathi's framework for CASE tool learnability, which is used throughout this paper. Section 3 discusses the research design used and the results of the study are provided in Section 4. The paper ends with a discussion in Section 5 and concluding remarks in Section 6.

\section{Background}

\subsection{Usability and learnability}

Different classification schemes for quantifying and assessing usability have been proposed (Gould and Lewis, 1985; Nielsen, 1993; Barnum, 2002; Dix et al., 2004; Seffah et al., 2006) and they all specify learnability as one of the key attributes of usability. The ISO/IEC 9126-1 definition of usability is concerned with the attributes of the software system that make it understandable, learnable, easy to use and attractive (ISO, 2001). In order to determine how easy it is to learn to use a CASE tool for students in diverse user communities, a framework for evaluating OO CASE tool learnability was required. The first framework investigated was one proposed by Phillips et al. for evaluating the usability of CASE tools (Phillips et al., 1998). 
The second framework reviewed was proposed by Senapathi and included only the learnability attributes of usability (Senapathi, 2005). Prior to the Phillips and Senapathi studies, methods and frameworks for evaluating CASE tools were mainly suitable for commercial environments (Mosley, 1992).

Since the focus of this study was on learnability, Senapathi's framework was selected as the most appropriate framework to use. An overview of this framework and the results of Senapathi's study is discussed in the next section.

\subsection{Senapathi's framework for evaluating CASE tool learnability}

A framework used to evaluate the learnability of CASE tools was designed by Senapathi based on the ISO 9241 definition of usability (Senapathi, 2005). This framework is illustrated in Figure 1. The framework proposes that the users' Satisfaction ratings of the Learnability attributes of a CASE tool are dependent on certain Context of use factors. These context of use or contextual factors include the tasks, the learning environment, the user characteristics and the CASE tool used.

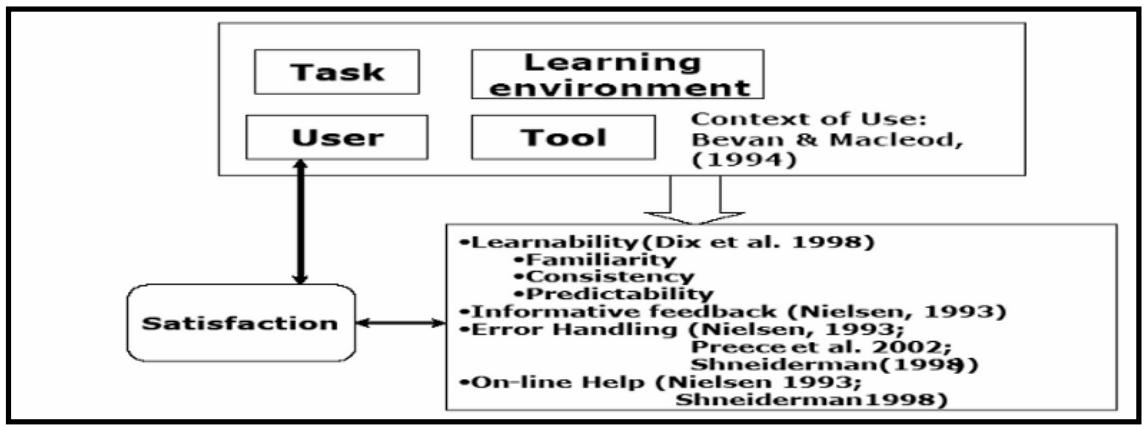

Figure 1 A framework for the evaluation of CASE tool learnability (Senapathi, 2005)

In an educational context, all the activities and assessments that require the use of a CASE tool are recognised as tasks (Senapathi, 2005). Senapathi's framework recognises the significance of the learning environment in the learnability evaluation of CASE tools in educational environments (Senapathi, 2005). The background and context in which the course is delivered should be studied and analysed. This includes the learning methodologies, teaching methods and resources used. CASE tools should be learnable in a short timeframe and support a wide range of different learner characteristics due to the time constraints of 
students (Senapathi, 2005). Senapathi's study explored the effects of five user characteristics on learnability. These were Gender; General level of computer experience; Previous experience with CASE tools; Number of hours spent per week with CASE tools; and Attitude and motivation.

The Tool aspect of the framework relates to the complexity of the selected CASE tool and how this complexity affects learnability (Senapathi, 2005). Senpathi's study used Rational Rose as the selected CASE tool. Satisfaction was used as a direct measure of evaluating whether or not a CASE tool is learnable in a given context (Senapathi, 2005).

Senapathi proposed six attributes that should be used to measure learnability: Familiarity, Consistency, Predictability, Informative Feedback, Error Handling and On-Line Help. Familiarity is the extent to which a user's knowledge and experience in other real-world or computer-based domains can be applied when interacting with a new system (Dix et al., 2004). Consistency is the likeness in behaviour arising from similar situations or similar task objectives (Dix et al., 2004). Predictability is the ability of the system to allow the user to anticipate the natural progression of each task. The presence of appropriate and relevant feedback, specifically in the context of handling error messages, is considered to have a significant effect on the learnability and understandability of a system (Jankowski, 1995; Norman, 1999). The quality of feedback provided by the CASE tool is an important learnability measure (Phillips et al., 1998). Human Computer Interaction (HCI) research highlights the importance of the provision of help facilities in software for improving usability and learnability (Nielsen, 1993; Post and Kagan, 2000; Senapathi, 2005; Seffah et al., 2006).

\subsection{Results and limitations of Senapathi's study}

The results of Senapathi's study showed that three of the five user characteristics tested had significant effects on learnability. These were Computer experience; Previous experience with CASE tools; and Attitude and motivation. The other two user characteristics showed no significant results. Senapathi's study also revealed that all groups rated the Error handling features of Rational Rose significantly lower than the other features, regardless of their user characteristics, specifically with regard to Consistency and Feedback (Senapathi, 2005).

Senapathi's study has several limitations. The first limitation is the fact that it was only tested at the University of Auckland, New Zealand and may not be suited for educational institutions with linguistically and technologically diverse communities where English is not the home language of the majority of the student population. The tool part of the framework was not verified as the study only included an evaluation of one CASE tool, namely Rational Rose.

The first section of the learnability questionnaire used by Senapathi included closed-ended questions that were grouped according to the learnability attributes 
in the framework. Ease of Learning, was not listed as a learnability attribute in Senapathi's original framework, but the first group of questions in Senapathi's learnability questionnaire were grouped under the heading, Ease of Learning. Ease of learning refers to the novice user's experience on the initial part of the learning curve while trying to learn a new system (Nielsen, 1993).

Although On-line help was listed as a learnability attribute in Senapathi's framework, no closed-ended questions relating to On-line help were included in the learnability questionnaire.

The next section discusses the research design for an experiment undertaken to validate Senapathi's framework at a South African university.

\section{Research design}

The primary research question of this study was to determine what CASE tool learnability problems are experienced by students in a diverse community. The second research question was to determine if there is a relationship between CASE tool learnability and the user's context of use. In order to answer these questions and to verify Senapathi's framework in a diverse user community, an experiment was performed in 2006. The participants of this experiment were students registered for an OOSAD course at a South African university, and were representative of the student population of that found in a typical university in South Africa.

The experiment included a usability evaluation of two CASE tools using the framework proposed by Senapathi. Two additional user characteristics were identified, namely home language and frequency of computer use (Section 1), which were not included in Senapathi's study. In order to verify the Tool section of the framework two CASE tools were selected, namely Microsoft Visio and IBM's Rational Software Modeller (a more recent version of Rational Rose). Microsoft Visio was selected as the least complex of the two CASE tools since it has fewer features than Rational Software Modeller (Scholtz, 2003).

\subsection{Hypotheses}

The null hypothesis " $H_{0}$ : No relationship exists between the learnability of a CASE tool and the context of use" was formulated for examination and tested for significance at the $95 \%$ significance level $(\alpha=.05)$. It was refined to produce the sub-hypotheses in Table 1. 
Table 1 Research Hypotheses

\begin{tabular}{|l|l|l|}
\hline \multicolumn{2}{|c|}{ Number } & Hypotheses \\
\hline $\mathrm{H}_{0.1}$ & & No learnability problems exist in either of the selected CASE tools. \\
\hline $\mathrm{H}_{0.2}$ & & $\begin{array}{l}\text { No relationship exists between the learnability of a CASE tool and the } \\
\text { type of tool used. }\end{array}$ \\
\hline $\mathrm{H}_{0.3}$ & $\begin{array}{l}\text { No relationship exists between the learnability of a CASE tool and the } \\
\text { user characteristics. }\end{array}$ \\
\hline & $\mathrm{H}_{0.3 .1}$ & $\begin{array}{l}\text { No relationship exists between the learnability of a CASE tool and the } \\
\text { user's home language. }\end{array}$ \\
\hline
\end{tabular}

\subsection{Evaluation instruments}

The post-test learnability questionnaire used in Senapathi's study was amended by adding a second questionnaire for the second CASE tool, which had an additional section at the end where the participant had to select his/her CASE tool preference as well as the reason for the preference. An attitude and motivation questionnaire was completed by all participants on completion of both tasks, where they were required to rate their attitude towards the use of a CASE tool in an OOSAD module. This questionnaire was the same as the one used in Senapathi's study.

\subsection{Participant and task selection}

Participants recruited for this experiment were students at a South African university enrolled for the Information Systems 2.1 module. Both groups were taught the same material on UML prior to the tasks and had no previous experience of CASE tools prior to starting the experiment. For Task A, the participants were required to draw a use case diagram, and for Task B, a class diagram. Sixty-two students agreed to participate in the study and stratified sampling was used to divide these students into two equivalent groups. Prior to 
commencing the first task, a background questionnaire was completed by each participant.

Most of the existing questionnaires, scales and tests that have been developed in highly industrialised countries of Europe and North America cannot be applied to the South African context without some modifications as the user communities in these countries are not as linguistically or technologically diverse as those in South Africa (Mouton, 2001).

Users who have different home languages and different frequency of computer use profiles may have different preferences for user interfaces and this could have an effect on the learnability of a CASE tool (Ford and Gelderblom, 2003; Shneiderman and Plaisant, 2005). Two additional user characteristics, namely, home language and frequency of computer use, were therefore added to the background questionnaire.

The profile of the participants according to home language and frequency of computer use is shown in Figure 2. Only $28 \%$ of the participants had English as their home language, and almost half $(46 \%)$ had a Low frequency profile (less than 10 hours per week computer use). The students were randomly assigned to each group, while making sure that the proportion of both gender and academic performance per group was representative of the actual student population. Each participant was required to complete both tasks in both CASE tools.
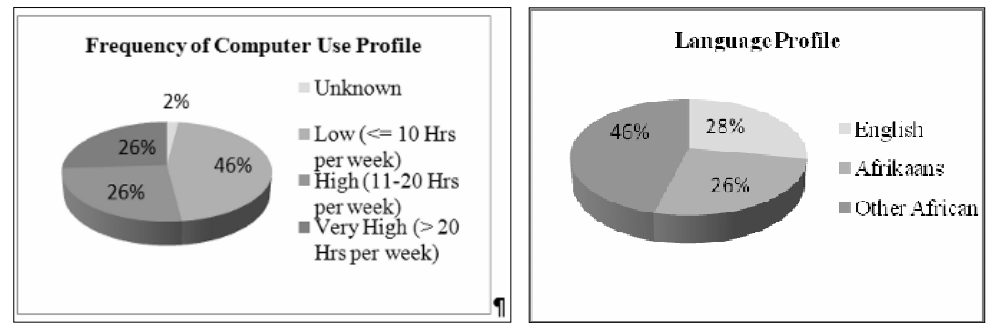

Figure 2 Profile of participants

The experimental design method used was a counterbalanced design and the same design was used for both tasks. The tasks were identical for both groups, except for the order of the CASE tools used. The one group used Visio first and the other group used Rational first. After each task, the participants completed a post-test learnability questionnaire for each CASE tool. 


\section{Research results}

Post-test questionnaires from only 46 of the original 62 participants could be included in the data analysis, as the remaining 16 participants did not attend both tasks.

\subsection{Quantitative results}

The first section of the post-test learnability questionnaire included 15 closedended items relating to the learnability attributes proposed in the framework and participants were asked to rate each item on a Likert scale of 1 to 5, where $1=$ "predominantly disagree" and 5="predominantly agree". Initial statistics on the responses from the first section of the learnability questionnaire revealed that Visio scored significantly higher than Rational for both tasks for all attributes except Familiarity. Figure 3 illustrates the mean satisfaction ratings for each learnability attribute for both tasks.

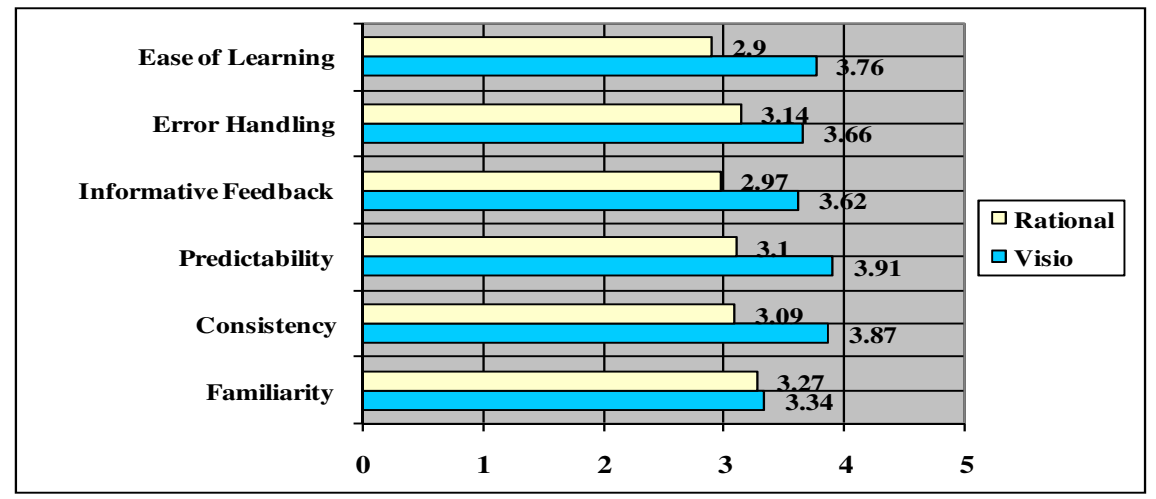

Figure 3 Learnability results for both tasks (5-point Likert scale)

The highest rated attribute for Rational for both tasks was Familiarity (with a mean rating of 3.27), whereas for Visio it was Predictability (with a mean rating of 3.91). The lowest rated attributes were Ease of learning for Rational (with a mean rating of 2.9) and Familiarity for Visio (with a mean rating of 3.34). From this we can deduce that the participants rated the two tools differently in terms of the six attributes of the CASE tools measured, and that the attributes that were rated best and worst for the two CASE tools were not the same. These results were obtained irrespective of whether the tool was used first or second. A repeated measures ANOVA test was conducted on the three factors and the overall mean ratings for 
the different learnability attributes. The first factor was the type of CASE tool used. The results of these tests showed that there is a definite relationship between the CASE tool used and learnability as the repeated measure for the tool used was significant for all of the attributes except Familiarity for both Task A and Task B. For both tasks, for all of the learnability attributes (except Familiarity), there was therefore an interaction in the mean ratings of both CASE tools from performing the task in the first tool and then in the second tool. Visio was rated higher than Rational irrespective of the order in which the tools were used.

The results of the ANOVA tests also revealed that a relationship exists between both of the user characteristics and learnability; namely home language and frequency of use. There was a significant interaction between the mean rating for Predictability for the Afrikaans language group and the African language group $(\mathrm{p}=.030)$. The mean rating for the Afrikaans language groups for Predictability dropped from the first CASE tool to the second CASE tool in Task A, whereas the African language group's mean rating increased. The mean rating for the English language group remained fairly constant from one tool to the other, which could be due to the fact that the language used in the user interface of both CASE tools is English. Frequency of computer use was significant for only one attribute, namely Error Handling, for both tasks. The interaction occurred between the Low ( $<=10$ hours per week) and the High (11-20 hours) frequency groups, for both tasks.

Visio was selected the preferred tool for Ease of Learning for both tasks by an overwhelming majority for Task A (78\%) and for Task B (72\%). The most frequent reason for selecting Visio as the preferred tool for Ease of Learning, was Simplicity. The participants were also asked to rate their attitude towards the use of a CASE tool in the OOSAD module. The mean rating per question is shown in Table 2 . 
Table 2 Attitude and motivation towards use of CASE tools in teaching OOSAD (5-point Likert scale)

\begin{tabular}{|l|l|l|l|l|}
\hline \multicolumn{1}{|c|}{ Question } & \multicolumn{1}{c|}{ Mean } & \multicolumn{1}{c|}{ SD } \\
\hline The use of a CASE tool for this course was a good idea. & 41 & 4.10 & 0.80 \\
\hline The CASE tool made my work more interesting & 41 & 3.71 & 0.81 \\
\hline $\begin{array}{l}\text { The use of a CASE tool enabled me to complete my tasks more } \\
\text { quickly. }\end{array}$ & 41 & 3.68 & 0.85 \\
\hline $\begin{array}{l}\text { The use of a CASE tool helped me to understand the underlying } \\
\text { concepts better. }\end{array}$ & 41 & 3.73 & 0.78 \\
\hline $\begin{array}{l}\text { Correct understanding and use of the CASE tool helped me to } \\
\text { perform better in the UML section of the course and assignments. }\end{array}$ & 41 & 3.80 & 0.78 \\
\hline Overall Attitude and Motivation & $\mathbf{4 1}$ & $\mathbf{3 . 8 0}$ & $\mathbf{0 . 6 8}$ \\
\hline
\end{tabular}

The results showed a favourable response towards using a CASE tool for teaching OOSAD and that using a CASE tool helped to understand the underlying UML concepts.

\subsection{Qualitative results}

The qualitative data collected from the responses to the open-ended questions were coded and structured into categories which were then matched to the corresponding learnability attribute. Frequency counts for each attribute were calculated in order to determine the most common attributes. The most frequent reason for selecting Visio as the preferred CASE tool for Ease of Learning, was Familiarity. One of the participants stated that Visio was "More familiar" and another that it was "Easier to learn and use since it has the same interface as Word and Excel".

Participants were also required to indicate what they did not like about each CASE tool and any problems encountered. Several learnability problems with both CASE tools were identified. For both tools the attribute with the highest frequency count for negative comments was Recoverability. One of the problems encountered by the participants was Visio's handling of erroneous associations between UML shapes. Visio highlights in red any part of the diagram which is in violation of UML rules, and displays the related error message in the error window below the drawing pane. Many of the students did not like this approach as they did not know why the association line was highlighted in red, due to the fact that the related error message was often not seen. One participant stated "The red lines indicate errors but these weren't obvious what they meant." Some of the students found Rational's approach to error prevention and handling very 
confusing as the CASE tool does not allow an erroneous association between shapes to be made. It does not display any message explaining why the association cannot be drawn, which led to frustration and confusion on the part of the student. One participant stated that "When you make a mistake the program does not allow you to perform the action at all. The program is confusing to use" Other participants made similar comments. The attribute that had the second most negative comments was Customisability for Visio. Most of the responses relating to this attribute were with regard to the inflexibility of the editing of the communication line names. The attribute with the most negative responses for Rational was Simplicity. It can be deduced from this that many of the participants found Rational to be too complex. This confirms the selection of this CASE tool as the more complex tool (Section 3).

\subsection{Hypotheses testing}

Several learnability problems were encountered by participants in both CASE tools, Microsoft Visio and Rational Software Modeller (Section 4.2), therefore $\mathrm{H}_{0.1}$ "No learnability problems exist in either of the selected CASE tools" can be rejected. As can be seen from the initial quantitative results reported (Section 4.1), the learnability ratings of the two CASE tools was significantly different for five of the six learnability attributes measured.

This was confirmed by the more detailed repeated measures ANOVA tests showing that the type of CASE tool used has an effect on learnability (Section 4.1). One can thus conclude that $\mathrm{H}_{0.2}$ "No relationship exists between the learnability of a CASE tool and the type of tool used" should also be rejected.

The statistics showed that there was a relationship between the home language of participants and some of the learnability attributes (Section 4.1), therefore the hypothesis $\mathrm{H}_{0.3 .1}$, "No relationship exists between the learnability of a CASE tool and the user's home language" was rejected. Initial results from the repeated ANOVA tests revealed that frequency of computer use was significant for one attribute, namely Error Handling, for both tasks (Section 4.1). The hypothesis $\mathrm{H}_{0.3 .2}$ 'No relationship exists between the learnability of a CASE tool and the user's frequency of computer use' was therefore rejected. Since both $H_{0.3 .1}$ and $H_{0.3 .2}$ were rejected, $H_{0.3}$ can be rejected. Since $H_{0.1}, H_{0.2}$ and $H_{0.3}$ can all be rejected, this implies that $\mathrm{H}_{0}$ "No relationship exists between the learnability of a CASE tool and the context of use" can be rejected. Table 4 summarises the research hypotheses and includes an indication whether the hypotheses can be rejected or not. 
Table 4 Summary of hypotheses and results

\begin{tabular}{|l|l|l|l|}
\hline \multicolumn{2}{|l|}{ Number } & \multicolumn{1}{|c|}{ Hypotheses } & Result \\
\hline $\mathrm{H}_{0.1}$ & & No learnability problems exist in either of the two CASE tools. & Rejected \\
\hline $\mathrm{H}_{0.2}$ & $\begin{array}{l}\text { No relationship exists between CASE tool learnability and the } \\
\text { type of tool used. }\end{array}$ & Rejected \\
\hline $\mathrm{H}_{0.3}$ & $\begin{array}{l}\text { No relationship exists between CASE tool learnability and the } \\
\text { user characteristics. }\end{array}$ & Rejected \\
\hline $\mathrm{H}_{0.3 .1}$ & $\begin{array}{l}\text { No relationship exists between CASE tool learnability and the } \\
\text { user's home language. }\end{array}$ & Rejected \\
\hline $\mathrm{H}_{0.3 .2}$ & $\begin{array}{l}\text { No relationship exists between CASE tool learnability and the } \\
\text { user's frequency of computer use. }\end{array}$ & Rejected \\
\hline
\end{tabular}

\section{Discussion}

One of the primary research questions of this study was to determine what problems students in a diverse community in South Africa experience with learning to use a CASE tool. The results of the experiment performed at a university in South Africa show that the majority of problems identified related to error prevention, error handling and feedback.

Microsoft Visio was clearly the preferred CASE tool for all the attributes of learnability. The attributes of learnability that were rated the highest by the students for their preferred CASE tool were Predictability, Consistency and Ease of learning. Designing CASE tools that are predictable, consistent and easier to learn can thus improve the rate of learning of CASE tools as well as indirectly improve the understanding of UML. The results of this study identified problems with the way in which both CASE tools tried to constrain UML errors, although the approaches used were very different. Visio's problems related to the way in which the erroneous UML connection was highlighted in red. Participants were not aware of the reason for this as the error message did not use language which was easy to understand and the message was displayed at the bottom of the screen. Rational uses error prevention and does not allow the participant to perform any erroneous UML connections. This frustrated the participants as they did not know why they were not allowed to perform the action.

One way of overcoming the problems with both of these approaches would be to use a confirmation error message when preventing an erroneous UML connection (Nielsen, 1994). The differences in users' reactions to the two approaches, as well as any proposed improvements in error handling, need further research before any conclusions can be made. A relationship was found to exist between the home language and frequency of computer use of the participants and their ratings of the two CASE tools, regardless of the order in which the tools were 
used. Further investigation with larger sample sizes is required in order to examine this relationship in more detail. Further research should be undertaken by extending the study to other universities in South Africa, which would provide a wider range of home language and frequency of computer use profiles. In order to be able to extend the results to commercial environments, similar evaluations could be undertaken at commercial organisations in South Africa.

\section{Conclusions}

This paper has investigated the learnability of CASE tools for education in a diverse student community at a university in South Africa. It discussed an experiment conducted at a South African university to address the limitations of Senapathi's framework in a South African context. The results of this research show that significant differences in learnability were found between the two CASE tools investigated. Relationships between two user characteristics and learnability were also identified, namely home language and frequency of computer use. The results also identified that simplicity is a key factor for learnability and that existing error handling techniques are problematic. These results can be used to improve the learnability of CASE tools and assist educators in selecting suitable CASE tools for diverse user communities.

\section{References}

Barnum, Carol (2002): Usability Testing and Research. Pearson. 0-205-31519-4. Booch, G, Rambaugh, J and Jacobson, I (1999): The Unified Modelling Language User Guide. Addison-Wesley.

Brewer, J and Lorenz, L (2003): Using UML and Agile Development Methodologies to Teach Object-Oriented Analysis \& Design Tools and Techniques. In Proceedings of CITC4'03, Lafayette, Indiana, USA:5457. ACM. October 16-18, 2003.

Burton, P and Bruhn, R (2004): Using UML to facilitate the teaching of objectoriented systems analysis and design. Journal of Computing Sciences in Colleges, 19(3):1937-4771. January 2004.

Dix, Allan, Finlay, Janet, Abowd, Gregory D and Beale, Russell (2004): HumanComputer Interaction. Third Edn, Prentice Hall.

Ford, Gabrielle and Gelderblom, Helene (2003): The Effects of Culture on Performance Achieved through the use of Human Computer Interaction. 
In Proceedings of SAICSIT 2003, Gauteng, South Africa. South African Institute for Computer Scientists and Information Technologists.

Frosch-Wilke (2003): Using UML in Software Requirements Analysis Experiences from Practical Student Project Work. In Proceedings of InSITE - Where Parallels Intersect. Informing Science. June.

Gould, J.D. and Lewis, C. (1985): Designing for usability: key principles and what designers think. Communications of the ACM, 28(3):300-311.

Greyling, Jean and Calitz, Andre (2003): The Development of a Computerised Multimedia Tutorial System for a Diverse Student Population. In Proceedings of 2nd International Conference on Computer Graphics, virtual reality, visualisation and interaction in Africa AFRIGRAPH '03, Cape Town, South Africa:109-116. ACM.

ISO (2001): ISO/IEC 9126-1: Software Product Evaluation [online]. Available at http://www.iso.org/iso/iso_catalogue/catalogue_tc/catalogue_detail.htm? csnumber $=22749$. [Accessed on July 2006].

Jankowski, DJ (1995): Case feedback in support of learning a systems development methodology. Journal of Information Systems Education, 7(3):88-90.

Jarzabek, S and Huang, R (1998): The Case for User-Centered CASE tools. Communications of the ACM, 41(8):93-99. August 1998.

Johnson, H.A and Wilkinson, L (2003): CASE tools in object-oriented analysis and design. In Proceedings of Eastern Conference: Consortium for Computing Services in Colleges.

Kemerer, Chris (1992): How the Learning Curve Affects CASE tool adoption. IEEE Software, 0740-0759/92/0500/0023. May 1992.

Koch, E (2002): Language Testing at UPE. In Proceedings of Language Testing Colloquium, UPE.

Lending, D and Chervany, N (1998): CASE tools: Understanding the Reasons for Non-Use. ACM SIGCPR conference on computer personnel research SIGCPR'98, 19(2). April 1998.

Mosley, V (1992): How to Assess Tools Efficiently and Quantitatively. IEEE Software, 9(3):29-32. May 1992.

Mouton, J (2001): How to succeed in your Master's \& Doctoral studies. Pretoria, Van Schaik Publishers.

Nielsen, J (1993): Usability engineering. Academic Press.

Nielsen, Jakob (1994): Ten Usability Heuristics [online]. Available at www.useit.com. [Accessed on February 2007].

Norman, D.A (1999): Affordance, conventions and design. In Proceedings of SIGCHI Interactions, 6(3):38-42. May 1999.

Phillips, C, Mehandjiska, D, Griffin, D, Choi, M.D and Page, D (1998): The usability component of a framework for the evaluation of OO CASE tools. In Proceedings of Software Engineering, Education and Practice, Dunedin:134-141. IEEE. 26 - 29 January 1998. 
Post, G and Kagan, A (2000): OO-CASE tools: an evaluation of Rose. Information and Software Technology, 42:383-388. 15 April 2000.

Rauchas, S, Rosman, B, Konidaris, G and Sanders, I (2006): Language Performance at High School and Success in First Year Computer Science. In Proceedings of SIGCSE '06, Houston, Texas, USA. ACM.

Scholtz, Brenda (2003): A Comparative Analysis of CASE Tools. Honours Treatise. Department of CS \& IS, University of Port Elizabeth. Port Elizabeth.

Seffah, Ahmed, Donyaee, Mohammed, Kline, Rex and Padda, Harkirat K (2006): Usability measurement and metrics:A consolidated model. Software Quality Journal, 14:159-178.

Senapathi, M (2005): A Framework for the Evaluation of CASE tool learnability in Educational Environments. Journal of Information Technology Education, 4.

Shneiderman, Ben and Plaisant, Catherine (2005): Designing the User Interface. Fourth Edn. 0-321-19786-0. 